\title{
Resistance to Extrinsic Stains by Hydrophobic Composite Resin Systems
}

\author{
W.H. DOUGLAS and R.G. CRAIG
}

University of Minnesota, Biomaterials Program, School of Dentistry, Minneapolis, Minnesota 55455 and University of Michigan, Department of Dental Materials, School of Dentistry, Ann Arbor, Michigan 48109

Measurement of color parameters demonstrated that hydrophobic composites had less staining capacity and greater ease of stain removal when compared with conventional composite materials. Accelerated aging of samples is important in staining tests.

J Dent Res 61(1):41-43, January 1982

\section{Introduction.}

It is likely that a number of factors contribute to the color change of composite resins in clinical service. Chemical instability of the resin system may lead to endogenous color change. Moreover, the ability to stain may be potentiated by surface conditions of the composite, such as the roughness or surface chemistry. The final factor to be considered is the resistance to removal of stain.

Some of these factors have been studied by a number of workers ${ }^{1,2}$ using a colorimeter to compare color differences for a number of resins and different staining techniques. Gross and Moser, ${ }^{3}$ using a four-filter colorimeter, studied the effects of dietary stains on four composites, and Moser et al., ${ }^{4}$ using the same stains and materials, assessed the tristimulus values, and transformed these into Munsell values, which are widely used in the visual perception of color. The quantification of color of composites has also been studied by Dennison et al., 5 using reflection spectrophotometry and Munsell color tabs. The efforts of these and many other workers have led to the application of good methods of color measurement in dentistry.

In previous communications, 6,7 the authors have reported on composites based on highly hydrophobic polymers, with respect to their physical and mechanical properties and resistance to microleakage. The purpose of the present report was to continue the study of the comparison between greater and lesser hydrophobic composites, with respect to staining capacity and ease of stain removal.

\section{Materials and methods.}

The materials used were two commercial composites, Adaptic ${ }^{*}$ and Concise, ${ }^{\dagger}$ which are good representatives of weakly hydrophobic (contact angle with water less than $\left.70^{\circ} \mathrm{C}\right)^{6}$ materials, and a candidate hydrophobic composite based on fluoromethacrylate (OFPMA indicates octafluoropentylmethacrylate), which has been reported in the literature. ${ }^{6,7}$ The experimental composite was $75 \%$ filled by weight with particulate quartz ( $50 \%$ of the filler had an equivalent spherical diameter less than $10 \mu \mathrm{m}){ }^{6}$ The commercial materials were mixed according to the

Received for publication November 10, 1980

Accepted for publication June 15, 1981

This investigation was supported in part by USPHS Research Grant DE-04050, from the National Institute of Dental Research, National Institutes of Health, Bethesda, MD 20205 and by the L.D. Caulk Co., Division of Dentsply International, Milford, DE 19963. This investigation was presented at the Annual Meeting of the A.A.D.R., Los Angeles, CA, March, 1980.

*Johnson and Johnson, New Brunswick, NJ 08520

$† 3 \mathrm{M}$ Manufacturing Company, St. Paul, MN 55101 manufacturers' instructions, and the candidate system was cured under visible light for $7.5 \mathrm{~min}$. The samples were prepared as discs, $40 \mathrm{~mm} \times 1.5 \mathrm{~mm}$ thick, against glass slides which produced an optically smooth surface. Thirtysix samples were prepared for each material, making a total of 108 samples in all. The study design is described in Table 1 .

TABLE 1

REPLICATION OF SAMPLES

\begin{tabular}{llccc}
\hline \hline & \multicolumn{4}{c}{ Material M } \\
\hline $\begin{array}{l}\text { Staining } \\
\text { Condition }\end{array}$ & 0 & 300 & 600 & 900 \\
\hline $\begin{array}{l}\text { No Stain } \\
\text { (Controls) }\end{array}$ & 3 & 3 & 3 & 3 \\
\hline Stained & 3 & 3 & 3 & 3 \\
\hline $\begin{array}{l}\text { Stained and } \\
\text { Washed }\end{array}$ & 3 & 3 & 3 & 3 \\
\hline
\end{tabular}

Aging procedure. - The samples for each material were randomly divided into four subgroups of nine samples each. Prior to staining, each subgroup was subjected to accelerated aging. 8 Aging was carried out in a weatherometer $\S$ under conditions of $43^{\circ} \mathrm{C}, 90 \%$ relative humidity, with an 18-minute water spray every $102 \mathrm{~min}$, and then subjected to irradiation from a 2400 xenon lamp. The times of exposure were zero, 300,600 , and $900 \mathrm{~h}$.

Staining procedure. - A staining procedure was developed based on the cationic dye methylene blue. This is a useful material since it will absorb directly onto silica. The ability of methylene blue to stain was further potentiated by the formation of a chlorhexidine complex. The clinical use of chlorhexidine mouthwashes can form a very retentive stain on human enamel and composites. ${ }^{9}$

A surface film was developed on the composites in the following manner: The samples were maintained in a vertical position by attachment to a glass slide in a Coplan jar. The samples were immersed in $25 \mathrm{ml}$ of a solution containing $0.2 \%$ chlorhexidine and 0.2 citric acid. To this solution was added $2 \mathrm{ml}$ of $2 \%$ sodium citrate and $2 \mathrm{ml}$ of $2 \%$ methylene blue dye in immediate succession. The jars were lightly shaken and allowed to stand for $24 \mathrm{~h}$.

This procedure immediately developed a finely divided precipitate of a chlorhexidine-citrate complex, which produced a visible blue-stained adhesive film on the surface of the composites (and on the optically smooth surface of a glass slide). After removal from the staining bath, excess fluid on the surface of the sample was removed by absorbent paper. A group of three samples from each of the aged groups of each material was left unstained as a control. All other samples were stained.

All samples, stained and unstained, were placed successively in a spectrophotometer ${ }^{\ddagger}$ with an integrating sphere

\footnotetext{
$\$$ Weather-Ometer 25 WR, Atlas Electric Devices, Chicago, IL 60613

‡CTA CIII, U-V Visible Spectrophotometer, Beckman Instruments, Inc., Irvine, CA 92664
} 
attachment, and a reflectance spectrum was derived for each with a standard black and a standard white background.

Stain removal. - Each set of stained samples (3) was placed in a $100-\mathrm{ml}$ beaker, aged side up, and $80 \mathrm{ml}$ of tap water were added. The beakers were then transferred to an ultrasonic cleaner (six at a time) and sonicated for five min. The samples were recovered, and excess water was absorbed by tissue paper; a reflectance curve was obtained for each sample, using a standard white and a standard black background.

Thus the staining test was multifactorial and included bulk staining, surface staining, the effect of aging, and the ease of stain removal.

Data reduction. - The reflectance curves were analyzed, and the CIE functions $X, Y$, and $Z$ were calculated. ${ }^{10}$ From these values, chromaticity coordinates $\mathrm{x}, \mathrm{y}$, and $\mathrm{z}$ were calculated, and reference to the chromaticity diagram gave the color parameters. These were the dominant wavelength, excitation purity, and luminous reflectance (or brightness). In addition, the contrast ratio (luminous reflectance on a black background compared to that on a white background) was calculated. The ratio gives a measure of the opacity of the specimen.

For each of the four color parameters, a three-way analysis of variance was carried out involving the material, $M$ ( 3 levels), the staining condition, $C$ ( 3 levels), and the time of aging, $\mathrm{T}$ (4 levels.). Scheffe intervals for multiple comparisons in all directions were calculated at $95 \%$ level of confidence.

\section{Results.}

Figs. 1-4 display the data as histograms. The bar height in each case represents the cell mean for that specific color parameter. Each Fig. shows three materials, three levels of staining condition, and, for brevity, two levels of aging time. The Scheffe intervals are as follows: dominant wavelength, 60; excitation purity, 0.29; luminous reflectance, 24 ; and contrast ratio, 0.14 . These values may be referred to in the histograms (Figs. $1-4$, respectively) for significant intervals.

A summary of some of the main effects is shown in Table 2, where the four levels of time of aging are combined, and two levels of staining condition and three

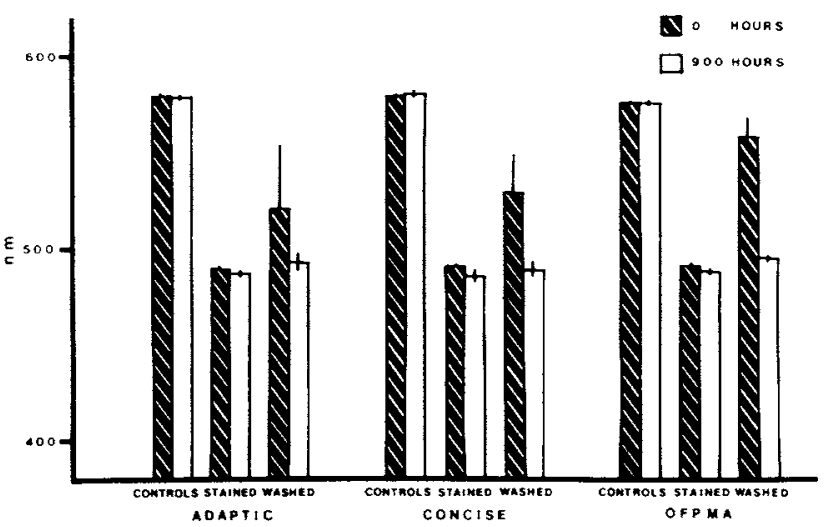

Fig. 1 - Dominant wavelength of stained and washed composites before and after aging.

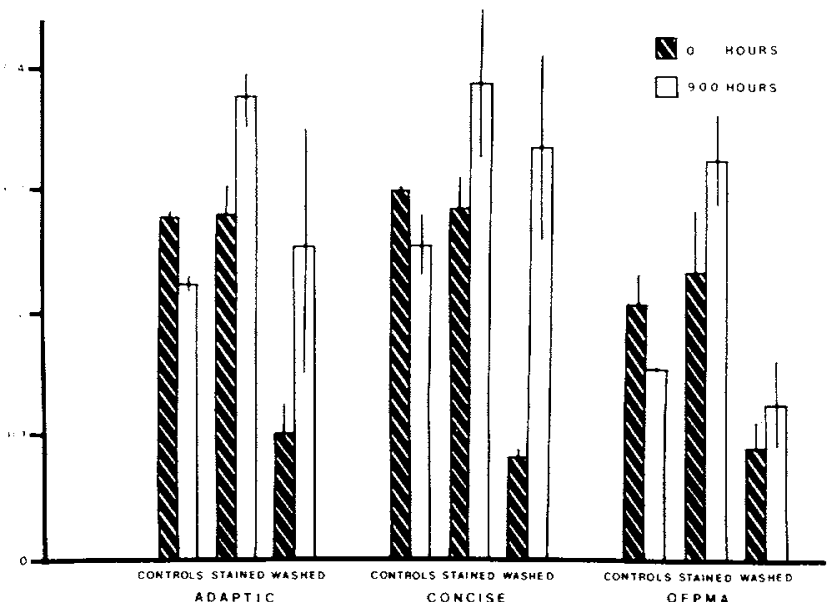

Fig. 2 - Excitation purity of stained and washed composites before and after aging.

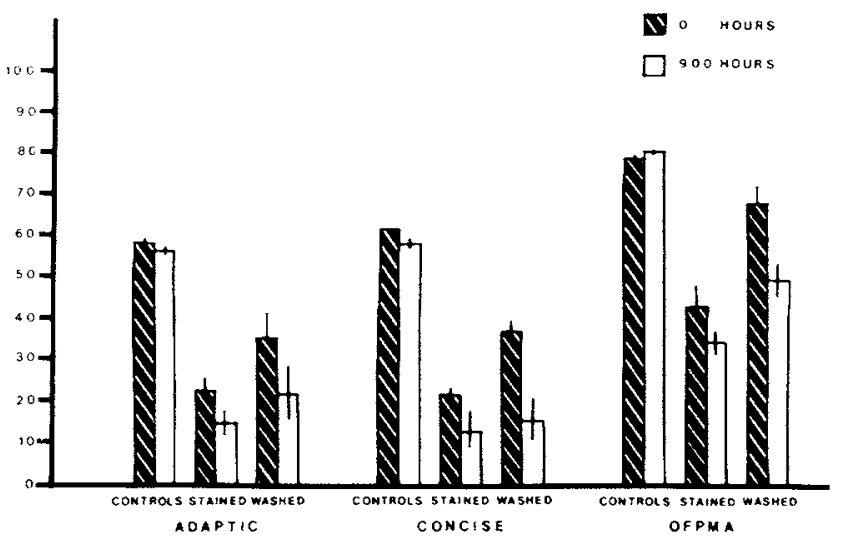

Fig. 3 - Luminous reflectance of stained and washed composites before and after aging.

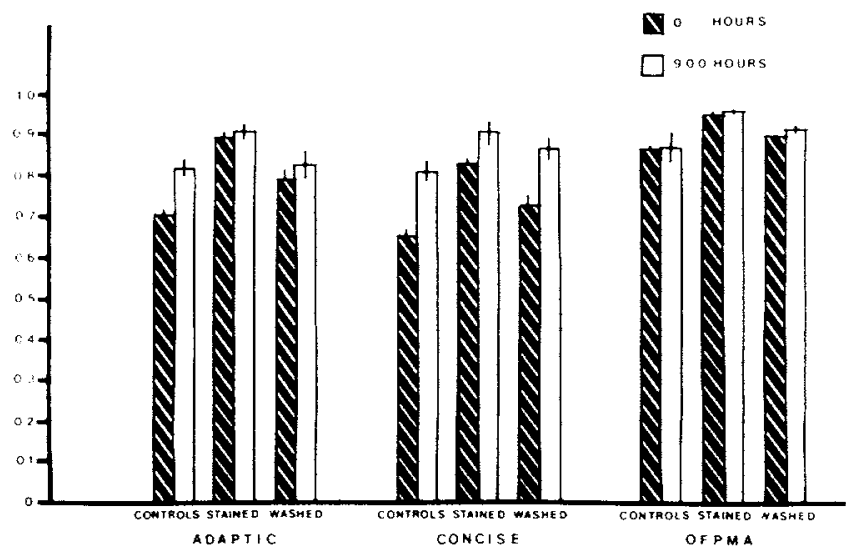

Fig. 4 - Contrast ratio of stained and washed composites before and after aging.

materials are compared. In Table 2 , the differences among the cell means are shown and not the cell means themselves.

\section{Discussion.}

Reference to the controls in Fig. 1 for the three materials shows that there is no change in the dominant wavelength of the color on aging. This is in agreement with Moser et al., 8 who found no change in the hue of the com- 
TABLE 2

SUMMARY OF THE MAIN EFFECTS SUMMED FOR THE FOUR LEVELS OF AGING (DIFFERENCES BETWEEN MEANS)

\begin{tabular}{|c|c|}
\hline $\begin{array}{ll}\text { Luminous } \mathrm{Re} \\
\mathrm{M}_{1} & 27.9 \\
\mathrm{M}_{2} & 31.9 \\
\mathrm{M}_{3} & 21.3\end{array}$ & $\begin{array}{l}1-C_{3} \\
\text { Scheffe }(0.95) 6.4\end{array}$ \\
\hline $\begin{array}{ll}\text { Dominant } W \\
\mathrm{M}_{1} & 71.3 \\
\mathrm{M}_{2} & 62.3 \\
\mathrm{M}_{3} & 52.1\end{array}$ & Scheffe 16 \\
\hline $\begin{array}{ll}\text { Excitation Pu } \\
\mathrm{M}_{1} & 0.109 \\
\mathrm{M}_{2} & 0.114 \\
\mathrm{M}_{3} & 0.0732\end{array}$ & $C_{3}$ \\
\hline $\begin{array}{ll}\text { Contrast Rati } \\
\mathrm{M}_{1} & 0.066 \\
\mathrm{M}_{2} & 0.075 \\
\mathrm{M}_{3} & 0.028\end{array}$ & Scheffe 0.037 \\
\hline
\end{tabular}

posites tested. In the stained condition, all the materials moved toward the blue-green color. The ability of the stained composites to recover their original color when washed showed important differences. The un-aged fluorocarbon showed the most recovery. However, with each material after $900 \mathrm{~h}$ of aging, there was almost no recovery in the wavelength toward that of the unstained controls.

As far as excitation purity is concerned, the controls showed (Fig. 2) that aging alone reduced the amount (or intensity) of the color in the unstained materials. In the stained condition, the aged samples took up a greater amount of color than did the un-aged. Furthermore, the histogram for the un-aged specimens (zero h) showed that, after washing, a considerable amount of the staining color was removed. This was not the case for the aged specimens $(900 \mathrm{~h})$, which retained a large amount of stain after washing. The exception is the fluorocarbon, which showed good recovery from the stain in both the aged and un-aged conditions.

With respect to luminous reflectance (Fig. 3), there was only a small difference in the controls which were aged, but not stained. Fig. 3 shows that, after staining, a considerable reduction in luminous reflectance of the specimens occurred. Some of the luminous reflectance of the specimens was recovered after washing - the greatest recovery again being found for the fluorocarbons.

A contrast ratio of 1.0 denotes a completely opaque specimen. Aging the specimens of Adaptic and Concise increased their opacity in each case (Fig. 4). This was not true with the fluorocarbon specimens, which already exhibited a high opacity. Staining the specimens made the opacity even higher, but there was good recovery in the un-aged specimens which had been washed. The recovery was less with the aged specimens - a trend which was consistent throughout the color parameters.
A summary of some of the main effects is shown in Table 2 , where the unstained control, $\mathrm{C}_{1}$, is compared with the stained and washed condition, $C_{3}$. This is totaled over the four levels of time of aging for the three materials. The numbers shown in Table 2 are the differences between the means and not the means themselves; they should be compared directly with the Scheffe intervals given.

In all cases, the differences for the fluorocarbon material $\left(\mathrm{M}_{3}\right)$ were fewer than those for Adaptic and Concise. Further, in excitation purity and contrast ratio, the differences for the fluorocarbon material were not statistically significant for $C_{1}-C_{3}$.

\section{Conclusions.}

The most obvious fact to emerge is the effect of aging on the staining performance of a composite. It appears advisable to age specimens before laboratory experiments are carried out on staining. In general, aged specimens stain more readily and are more resistant to hygiene procedures. The fluorocarbon specimens that had been aged, however, showed good recovery after ultrasonic cleaning. This may be a result of the fact that fluorocarbons resist the effect of aging at high humidity.

The original interest in the fluorocarbon polymers as a matrix for composites was in their ability to resist microleakage; however, the results of this study suggest that their ability to resist extrinsic stains may also be an important asset.

\section{REFERENCES}

1. KAFALIAS, M.C.; SWARTZ, M.L.; and PHILLIPS, R.W.: Physical Properties of Selected Dental Resins. Part I, J Prosthet Dent 13:1087-1107, 1963 .

2. PETERSON, E.A.; PHILLIPS, R.W.; and SWARTZ, M.L.: A Comparison of the Physical Properties of Four Restorative Resins, JADA 73:1324-1336, 1966.

3. GROSS, M.D. and MOSER, J.B.: A Colorimetric Study of Coffec and Tea Staining of Four Composite Resins, J Oral Rehab 4:311-322, 1977.

4. MOSER, J.B.; WOZNIAK, W.T.; MULLER, T.P.; and MOORE, B.K.: Use of the Munsell System to Compute Color Differences in Composite Resins, $J$ Dent Res 57:958-963, 1978.

5. DENNISON, J.B.; POWERS, J.M.; and KORAN, A.: A Color of Dental Restorative Resins, $J$ Dent Res 57:557-562, 1978.

6. DOUGLAS, W.H.; CRAIG, R.G.; and CHEN, C.J.: A New Composite Restorative Based on a Hydrophobic Matrix, $J$ Dent Res 58:1981-1986, 1979.

7. DOUGLAS, W.H.; CHEN, C.J.; and CRAIG, R.G.: Neutron Activation Analysis of Microleakage Around a Hydrophobic Composite, J Dent Res 59:1507-1510, 1980.

8. POWERS, J.M.; DENNISON, J.B.; and KORAN, A.: Color Stability of Restorative Resins Under Accelerated Aging, $J$ Dent Res 57:964-970, 1978.

9. CIANCIO, S.G.: Dental Therapeutics in Periodontics, Dent Clin North Am 24:814, No. 4, 1980.

10. WYSZECKI, G. and STILES, W.S.: Color Science, New York: John Wiley \& Sons, 1967, p. 628. 